\section{§19. Temperature Gradient Driven Short Wavelength Modes in Sheared Slab Plasmas}

Gao Zhe (Tsinghua Univ., China)

Sanuki, H., Itoh, K.

Dong, J.Q. (SWIP, China)

Understanding the anomalous transport in magnetically confined plasmas has been one of the major challenges for magnetic confinement fusion research for decades. It is now widely accepted that the anomalous transport is induced by turbulent fluctuations with small scales, the so-called microinstabilities. In particular, the temperature gradient (TG) driven instabilities are proposed as the plausible candidates responsible for anomalous thermal transport and have been studied extensively. Recent experiments have shown that the ion thermal diffusivity reduces to the neoclassical level in improved confinement tokamak plasmas.

The electron temperature gradient (ETG) mode in the electron diamagnetic direction is unstable in the very short wavelength regime. An unstable mode driven by temperature gradients in the short wavelength regime was recently identified by Smolyakov et al. Also, Hirose at al. indicate the existence of a temperature gradient mode in the short wavelength regime by using a kinetic electromagnetic integral code.
In this work, we confurm the explanation associated with the growth rate hump structure in the short wavelength regime discussed by $\mathrm{Pu}$ and Migliulo, showing that the local short wavelength mode is just attributable to the Landau damping/inverse Landau damping mechanism and the nonmonotonic behaviors of the real frequency as the wavelength varies. We also find that the electron kinetics strongly influences the properties of the mode in the short wavelength regime.

One of the results discussed in the present studies is shown in Fig.1. Shown in Fig.1 the growth rates of Short wavelength fundamental mode (S0) and first harmonic mode (S1) as functions of $\beta_{e}$. Although the S1 mode can be stabilized by bate effect but the SI mode hardly is stabilized by the beta effect.

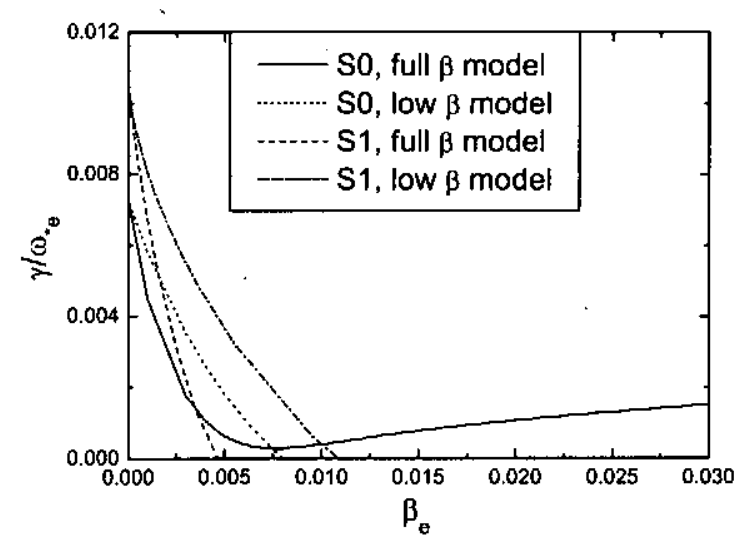

Fig.1 Normalized growth rate as function of $\beta_{e}$.

Reference: Zhe gao, H. Sanuki, K. Iton and J. Q.J Dong, Phys. Plasmas 10(2003) 2831. 